\title{
Accuracy of Sentinel Lymph Node Biopsy in Breast Cancer: Pitfalls in the Application of Single Tracers
}

\author{
Jiqiao Yang ${ }^{1,2}$ \\ $\mathrm{Li} \mathrm{Xu}^{\prime}$ \\ Pengcheng Liu' \\ Zhenggui $\mathrm{Du}^{\prime}$ \\ Jie Chen' \\ Faqing Liang' \\ Quanyi Long' \\ Di Zhang ${ }^{1,3}$ \\ Helin Zeng' \\ Qing Lv'
}

'Department of Breast Surgery, West China Hospital, Sichuan University, Chengdu, People's Republic of China; ${ }^{2}$ Clinical Research Center for Breast, West China Hospital, Sichuan University, Chengdu, People's Republic of China;

${ }^{3}$ Cancer Research Institute, Kanazawa University, Kanazawa, Japan
Correspondence: Qing Lv

Department of Breast Surgery, West

China Hospital, Sichuan University,

Guoxuexiang 37, Chengdu 61004I,

People's Republic of China

Tel +86-2885422873

Email Ivqingwestchina@163.com
This article was published in the following Dove Press journal: Cancer Management and Research

Background: Radioisotopes and blue dyes are used as dual tracers in the current gold standard procedure of sentinel lymph node (SLN) biopsy (SLNB) performed for breast cancer. However, the blue dye or the radioisotope as a single tracer is also being applied in some institutes. We aimed to explore the risk factors for the miss-detection of SLNs with the radioisotope and the blue dye and to describe the distribution of SLNs missed by each tracer.

Patients and Methods: Patients undergoing SLNB with radioisotope and blue dye as dual mapping agents were enrolled between August 2010 and August 2018. Radioactivity count, blue dye staining status, and size and location of each SLN were prospectively documented.

Results: In total, 2382 SLNs from 1010 patients were included for statistical analyses. The sentinel node identification rate was $100 \%$ for dual tracers, $99.4 \%$ for radioisotope, and $89.1 \%$ for blue dye. SLN identification using the blue dye was more likely to fail in patients undergoing breast-conserving surgery $(p<0.001)$ and mastectomy with reconstruction $(p=0.005)$. Furthermore, miss-detection was significantly more frequent in smaller and uninvolved nodes. Among all SLNs, 8.2\% were located in level II and one was in level III. Notably, single tracer of blue dye tended to fail in the detection of lymph nodes in higher levels $(p<0.001)$.

Conclusion: This study explored the association between features and the incidence of the failure to detect SLNs using radioisotope and blue dye. The locations of the miss-detected SLNs are demonstrated to provide a reference for SLNBs conducted using blue dye or radioisotope as a single tracer.

Keywords: breast cancer, sentinel lymph node, radioisotope, blue dye, single tracer

\section{Introduction}

Since axillary lymph node dissection (ALND) was replaced by sentinel lymph node (SLN) biopsy (SLNB) for axillary staging in patients with early-stage breast cancer, ${ }^{1,2}$ several tracing methods for SLNB have evolved, including radioisotope technetium$99 \mathrm{~m}$ sulfur colloid $\left({ }^{99 \mathrm{~m}} \mathrm{Tc}\right){ }^{3}$ blue dye, ${ }^{4,5}$ indocyanine green fluorescence, ${ }^{4,5}$ magnetic tracer, ${ }^{6-8}$ and carbon nanoparticles ${ }^{5}$ as well as clips. ${ }^{9}$ With identification rates of 96-97\% in large trials, ${ }^{10,11}$ the radioisotope and the blue dye combination remains the most widely accepted, long-established approach and the current gold standard for SLNB. ${ }^{12,13}$ However, given the limited accessibility to radioisotope and extra requirements for equipment, SLNBs with single tracer, especially blue dye are being adopted in a growing number of institutes, predominantly in less developed areas. ${ }^{14}$ Additionally, blue dye use has been rejected in some institutions because of 
complications such as anaphylaxis after application of the patent blue $\mathrm{V} ;{ }^{15}$ several studies that omitted the blue dye use do not necessarily report a poor identification rate or a high false-negative rate under certain conditions. ${ }^{16}$

This study aimed to assess in depth the pattern of SLN miss-detection with radioisotope and/or blue dye as tracers. Currently, in the majority of existing studies on SLNB, the subjects were individual patients. Previously, studies on single tracers were mainly carried out in two ways: 1) single-arm studies comparing the results of one tracer with existing data and 2) studies comparing two single tracers in different patients, with one group of patients undergoing SLNB with one tracer and a different group undergoing SLNB with another tracer. Distinctively, the current study prospectively documented the staining status of lymph nodes and lymphatic vessels, enrichment of nuclides, and the precise location of each lymph node retrieved during surgery, making it possible to analyze the efficacy of SLNB with radioisotope and blue dye, respectively, in a same group of patients, to perform node-targeted statistical analyses, and to describe the miss-detect pattern for the radioisotope and the blue dye. This information is of considerable importance in clinical practice.

\section{Patients and Methods}

\section{Patients}

Patients undergoing SLNB were enrolled in this prospective study between August 2010 and August 2018. Patients with pathologically diagnosed invasive breast cancer who were clinically node negative (negative on ultrasound, ${ }^{17}$ mammography, and physical examination) and those who underwent mastectomy with pathologically diagnosed ductal carcinoma in situ were eligible. Patients with clinically positive axillary lymph nodes, those with recurrence or distant metastasis, and those with prior axillary surgeries were excluded.

\section{Surgical Management}

We applied two mapping agents for SLNB. The radioisotope $\left({ }^{99 \mathrm{~m}} \mathrm{Tc}\right)$ was injected $2-18 \mathrm{~h}$ before surgery, and the blue dye (methylene blue) was injected 15-20 min before surgery. The radioisotope was injected intradermally at the tumor surface and/or at the periareolar site. For the blue dye, intradermal/subcutaneous injections at the tumor surface and/or at the periareolar site were adopted, based on whether the nipple-areola complex or skin at the tumor surface was conserved during surgery.
During surgery, a gamma probe of ${ }^{99 \mathrm{~m}} \mathrm{Tc}$ was used to identify SLNs. Any nodes with $10 \%$ or more of the ex vivo count of the hottest node in a patient and any node with at least one blue afferent lymphatic vessel derived from the breast were removed and designated as SLNs. ${ }^{18}$ Suspicious lymph nodes, which were firm, enlarged, and palpable, were removed and sent for pathological testing as non-SLNs. All SLNs were sent as frozen sections, and ALND was performed based on the pathological evaluation of the intraoperative frozen sections. Generally, patients with a failed SLNB and those with SLN macrometastases underwent ALND. Patients with SLNs with isolated tumor cells or metastasis-free SLNs were excluded from further axillary intervention. For SLNs with micrometastases, the decision to perform axillary dissection was made jointly by the patient and the surgery group before surgery.

We prospectively documented the radioactivity count, the status of blue dye staining of the node and lymphatic vessels, and the size and location of each SLN. To describe the distribution of SLNs, we classified the documented locations as level III, level II, and level I, with level I further classified as follows: zone A, the area around the margin of the breast tissue and the tail of Spence; zone $\mathrm{B}$, the area between the lateral margin of the pectoralis major and the thoracodorsal vessels; and zone $\mathrm{C}$, the lateral portion of the axilla. The majority of SLNs were in level I zones A and B. Additionally, we cautiously archived the demographic, clinical, and pathological data of each patient. The criteria for pathological reports were published previously. ${ }^{19}$

\section{Statistical Analysis}

For data analysis, negative intraoperative pathological reports of SLNs in combination with confirmed positive postoperative reports of SLNs, non-SLNs, and/or any nodes from ALND were defined as false-negative results.

Clinical and pathological features among groups were compared using one-way analysis of variance, Pearson's chi-square test, or Fisher's exact test for categorical data and independent $t$-tests for continuous data. The McNemar chi-square was used to compare the efficacy of the two tracers in SLNs of different locations. Furthermore, logistic regression with a backward stepwise model was used to identify risk variables for missed detection and falsenegative events, with $p$-values reported from the Wald tests and $95 \%$ confidence interval (CI) for the odds ratio (OR). All tests were two-tailed, and the significance level 
was 0.05. Statistical analysis was performed using SPSS 16.0 (SPSS Inc., Chicago, IL, USA).

\section{Results}

\section{Patient Information}

SLNB was successfully performed in 1011 patients. In one patient, a single radioisotope tracer was used because of a suspected anaphylaxis reaction to methylene blue; thus, this patient was excluded. Therefore, 2382 SLNs from 1010 patients who underwent SLNB with dual tracers were included in the data analysis. The clinicopathologic features of the included patients are listed in Table 1.

\section{Incidence of Missed Detection and False-Negative Events}

Among the patients, the overall rate of axillary involvement was $32.6 \%(329 / 1010)$. The sentinel node identification rate was $100 \%(1010 / 1010)$ for dual tracers, $99.4 \%$ (1004/1010) for radioisotope only, and $89.1 \%$ (900/1010) for blue dye only. The identification rate of blue dye only was significantly lower than that of radioisotope only $(p<0.001)$ and that of dual tracers $(p<0.001)$. We explored the risk indicators for the detection failure of blue dye. Breast-conserving surgery rather than mastectomy and reconstruction was risk indicators for detection failure, whereas prior excisional biopsy and number of SLNs $\geq 3$ were protective indicators (Table 2). However, none of the indicators for detection failure for radioisotopes were identified because of the small number of failed cases.

The false-negative rate was $3.8 \%$ (38/1010) for dual tracers, $5.7 \%$ (58/1010) for radioisotope only, and 7.2\% (73/1010) for blue dye only. The false-negative rate of blue dye only was significantly higher than that of dual tracers $(p=0.001)$. Logistic regression was employed to identify the risk indicators for false-negative events. The number of SLNs $\geq 3$ was the only protective indicator for false-negative events with combined tracers (OR $[95 \% \mathrm{CI}]$ $=0.150[0.043-0.518], p=0.003)$. However, none of the indicators for false-negative events with a single tracer were identified.

\section{Lymph Node-Specific Risk Factors for Missed Detection}

Among the 2382 SLNs, 1243 (52.2\%) were detected by both the radioisotope and the blue dye, $741(31.1 \%)$ were detected by radioisotope only, and 398 (16.7\%) were detected by blue dye only.
Table I Clinicopathological Characteristics of Included Patients $(n=1010)$

\begin{tabular}{|c|c|c|}
\hline Variable & $\mathbf{n}$ & Percentage \\
\hline Age (mean $\pm S D)$ & \multicolumn{2}{|c|}{$48.28 \pm 11.30$} \\
\hline \multicolumn{3}{|l|}{ Type of surgery } \\
\hline Mastectomy & 846 & $83.8 \%$ \\
\hline Breast-conserving surgery & 164 & $16.2 \%$ \\
\hline \multicolumn{3}{|l|}{ Reconstruction after mastectomy } \\
\hline Yes & 145 & $14.4 \%$ \\
\hline No & 865 & $85.6 \%$ \\
\hline \multicolumn{3}{|l|}{ PT classification } \\
\hline Tis-TI & 588 & $58.2 \%$ \\
\hline $\mathrm{T} 2-4$ & $34 I$ & $33.8 \%$ \\
\hline Unknown & 81 & $8.0 \%$ \\
\hline \multicolumn{3}{|l|}{ Axillary involvement } \\
\hline Yes & 329 & $32.6 \%$ \\
\hline No & 681 & $67.4 \%$ \\
\hline \multicolumn{3}{|l|}{ Excision biopsy before surgery } \\
\hline Yes & 207 & $20.5 \%$ \\
\hline No & 803 & $79.5 \%$ \\
\hline \multicolumn{3}{|l|}{ Pathological diagnosis } \\
\hline DCIS and Paget's disease & 112 & $11.1 \%$ \\
\hline Invasive ductal carcinoma & 790 & $78.2 \%$ \\
\hline Others $^{\mathrm{a}}$ & 108 & $10.7 \%$ \\
\hline \multicolumn{3}{|l|}{ Tumor Location } \\
\hline Upper outer quadrant & 362 & $35.8 \%$ \\
\hline Other quadrants & 574 & $56.8 \%$ \\
\hline Unknown & 74 & $7.3 \%$ \\
\hline \multicolumn{3}{|l|}{ Number of SLNs detected } \\
\hline $\mathrm{I}-2$ & 624 & $61.8 \%$ \\
\hline $3-4$ & 334 & $33.1 \%$ \\
\hline$\geq 5$ & 52 & $5.1 \%$ \\
\hline
\end{tabular}

Notes: Including papillary carcinoma, mucous carcinoma, invasive lobular carcinoma, malignant phyllode tumor, secretory carcinoma, metaplastic carcinoma, squamous cell carcinoma, adenoid cystic carcinoma and mixed carcinoma.

Abbreviations: SD, standard deviation; SLNs, sentinel lymph nodes; DCIS, ductal carcinoma in situ.

We compared the lymph node-specific characteristics between SLNs that were identified and missed by each tracer. Independent of the tracing method, the missdetected SLNs were significantly smaller in size than those successfully detected ( $p<0.001$ for both tracers). Moreover, nodes with tumor metastasis were more likely to be successfully identified than negative lymph nodes ( $p=0.014$ for radioisotope and $p<0.001$ for blue dye). In terms of location, single tracer of blue dye tended to fail in the detection of SLNs at higher levels $(p<0.001)$. However, such a phenomenon did not occur in the setting 
Table 2 Logistic Regression of Predictors for Detection Failure of Single Tracer of Blue Dye

\begin{tabular}{|c|c|c|c|}
\hline Variable & OR & $95 \% \mathrm{Cl}$ & $p$-value \\
\hline \multicolumn{4}{|l|}{ Age } \\
\hline$>35$ & 1.00 & & \\
\hline$\leq 35$ & 1.202 & $(0.601-2.403)$ & 0.604 \\
\hline \multicolumn{4}{|l|}{ Type of surgery } \\
\hline Mastectomy & 1.00 & & \\
\hline Breast-conserving surgery & 2.525 & $(1.483-4.3)$ & $0.00 I^{*}$ \\
\hline \multicolumn{4}{|l|}{ Reconstruction } \\
\hline No & 1.00 & & \\
\hline Yes & 2.367 & $(1.289-4.347)$ & $0.005^{*}$ \\
\hline \multicolumn{4}{|l|}{ PT classification } \\
\hline Tis-TI & 1.00 & & \\
\hline $\mathrm{T} 2-4$ & 0.700 & $(0.430-1.14 I)$ & 0.152 \\
\hline \multicolumn{4}{|l|}{ Axillary involvement } \\
\hline No & 1.00 & & \\
\hline Yes & 0.821 & $(0.505-1.336)$ & 0.427 \\
\hline \multicolumn{4}{|l|}{ Excision biopsy before surgery } \\
\hline No & 1.00 & & \\
\hline Yes & 0.405 & $(0.170-0.964)$ & $0.04 I^{*}$ \\
\hline \multicolumn{4}{|l|}{ Tumor location } \\
\hline Upper outer quadrant & 1.00 & & \\
\hline Other quadrants & 1.569 & $(0.974-2.528)$ & 0.064 \\
\hline \multicolumn{4}{|l|}{ Number of SLNs retrieved } \\
\hline $\mathrm{I}-2$ & 1.00 & & \\
\hline$\geq 3$ & 0.606 & $(0.369-0.996)$ & $0.048^{*}$ \\
\hline
\end{tabular}

Note: ${ }^{*} p<0.05$

Abbreviations: OR, odds ratio; $\mathrm{Cl}$, confidence interval; SLNs, sentinel lymph nodes.

of radioisotope. Moreover, we explored whether discrepancies existed between different sites of blue dye injection, but there were no significant differences (Table 3). Notably, among the SLNs with tumor cell involvement missed by the blue dye, $14.1 \%(12 / 85)$ were located in level II.

\section{Distribution of SLNs and Locations of Miss-Detected SLNs}

The precise location of 2364 out of 2382 SLNs was described. The location of each SLN was summarized according to whether it was missed by the radioisotope or the blue dye (Table 4). Among the 2364 SLNs, one $(0.04 \%)$ was in axillary level III and 196 (8.3\%) were in level II.

McNemar test revealed that the efficacy of the radioisotope and the blue dye as single tracers was different in
SLNs of level II and level I zone B. More specifically, for SLNs in level II, $58.7 \%(115 / 196)$ were missed by the blue dye and only $14.3 \%$ (28/196) were missed by the radioisotope. For those in level I zone B, 32.9\% (456/1388) were missed by the blue dye and $17.2 \%$ (239/1388) were missed by the radioisotope. Moreover, the only SLN identified in level III was successfully detected by the radioisotope but missed by the blue dye (Table 4).

\section{Discussion}

After the publication of the ACOSOG Z0011 ${ }^{20}$ and the diffusion of the American Society of Clinical Oncology clinical practice guidelines ${ }^{21}$ pointing out the futility of lymphadenectomy even in the presence of metastases in the SLN, the traditional concept of axillary dissection in breast cancer is being questioned. However, while the emerging role of axillary ultrasound in the preoperative staging of the axilla ${ }^{22}$ and in the follow-up after SLNB ${ }^{23}$ and the possibility of avoiding SLNB are currently being evaluated in a prospective study, ${ }^{24}$ SLNB remains the gold standard for the axillary management of early-stage breast cancers. In this study, we prospectively recorded the features of 1010 patients who successfully underwent SLNB using tracers of radioactive ${ }^{99 \mathrm{~m}} \mathrm{Tc}$ and blue dye to explore the differences among tracing methods of radioisotope only, blue dye only, and dual tracers in the same individuals. In this study, the identification rates of dual tracers $(100 \%)$ and single radioisotope tracer (99.4\%) were high, but that for blue dye used as a single tracer $(89.1 \%)$ was $<90 \%$. In addition, the false-negative rates of both tracers used as single tracers were higher than 5\% (5.7\% for radioisotope and $7.2 \%$ for blue dye), indicating that SLNB with single tracer of radioisotope or blue dye should be applied with extra caution in patients with breast cancer.

An analysis of the indicators for missed detection using a single tracer revealed that breast-conserving surgery, rather than mastectomy, was a risk indicator for blue dye. This might be explained by the minimal incision of the SLNB for the sake of esthetics, resulting in limited visualization and thus hampering the detection and identification of SLNs. A similar interpretation also applies to the fact that SLN identification using only the blue dye was more likely to fail in patients who underwent reconstruction after mastectomy. To preserve the appearance of the breast as much as possible, skin- and nipple-sparing mastectomies are usually performed to allow for subsequent reconstruction. When the incision was made at the upper or lateral portion of the breast 
Table 3 Associations Between Node-Specific Features and Incidence of Miss-Detection by Radioisotope and Blue Dye, Respectively

\begin{tabular}{|c|c|c|c|c|c|c|c|}
\hline Variable & $\begin{array}{l}\text { Overall } \\
(n=2382)\end{array}$ & $\begin{array}{l}\text { SLNs Detected by } \\
\text { Radioisotope } \\
(n=1984)\end{array}$ & $\begin{array}{l}\text { SLNs Missed by } \\
\text { Radioisotope } \\
(n=398)\end{array}$ & $p$-value & $\begin{array}{l}\text { SLNs Detected } \\
\text { by Blue Dye } \\
(n=1641)\end{array}$ & $\begin{array}{l}\text { SLNs Missed by } \\
\text { Blue Dye } \\
(n=741)\end{array}$ & $p$-value \\
\hline $\begin{array}{l}\text { Size of lymph node } \\
\text { (mean } \pm \mathrm{SD})(\mathrm{mm})\end{array}$ & $10.68 \pm 7.34$ & $11.16 \pm 7.47$ & $8.28 \pm 6.11$ & $<0.001 *$ & $|1.4| \pm 7.72$ & $9.02 \pm 6.07$ & $<0.001 *$ \\
\hline $\begin{array}{l}\text { Involvement of lymph node } \\
\text { Negative } \\
\text { Positive }\end{array}$ & $\begin{array}{l}2003 \text { (84.1\%) } \\
379(15.9 \%)\end{array}$ & $\begin{array}{l}1652(83.3 \%) \\
332(16.7 \%)\end{array}$ & $\begin{array}{l}35 \mathrm{I}(86.9 \%) \\
47(13.1 \%)\end{array}$ & $0.014^{*}$ & $\begin{array}{l}1347(82.1 \%) \\
294(17.9 \%)\end{array}$ & $\begin{array}{l}656(88.5 \%) \\
85(11.5 \%)\end{array}$ & $<0.001 *$ \\
\hline $\begin{array}{l}\text { Location of lymph node } \\
\text { Level I } \\
\text { Level II III }\end{array}$ & $\begin{array}{l}2169(91.1 \%) \\
197(8.3 \%)\end{array}$ & $\begin{array}{l}1803(90.9 \%) \\
169(8.5 \%)\end{array}$ & $\begin{array}{l}366(92.0 \%) \\
28(7.0 \%)\end{array}$ & 0.337 & $\begin{array}{l}\text { I5 } 548(94.3 \%) \\
81 \text { (4.9\%) }\end{array}$ & $\begin{array}{l}621(83.8 \%) \\
116(15.7 \%)\end{array}$ & $<0.001 *$ \\
\hline $\begin{array}{l}\text { Injection site } \\
\text { Tumor site } \\
\text { Periareolar } \\
\text { Tumor site and periareolar }\end{array}$ & $\begin{array}{l}1941(81.5 \%) \\
55(2.3 \%) \\
52(2.2 \%)\end{array}$ & & & & $\begin{array}{l}1305(79.5 \%) \\
40(2.4 \%) \\
40(2.4 \%)\end{array}$ & $\begin{array}{l}636(85.8 \%) \\
15(2.0 \%) \\
12(1.6 \%)\end{array}$ & 0.241 \\
\hline
\end{tabular}

Notes: a Only one SLN of level III was detected; ${ }^{*} p<0.05$.

Abbreviations: SD, standard deviation; SLNs, sentinel lymph nodes.

Table 4 The Locations of SLNs Divided by Whether Detected by Radioisotope or Blue Dye

\begin{tabular}{|c|c|c|c|c|c|c|c|c|c|c|c|}
\hline \multirow[t]{2}{*}{ Location } & \multicolumn{2}{|l|}{$\begin{array}{l}\text { Overall } \\
(n=2364)\end{array}$} & \multicolumn{2}{|c|}{$\begin{array}{l}\text { SLNs Detected by } \\
\text { Radioisotope } \\
(n=1970)\end{array}$} & \multicolumn{2}{|c|}{$\begin{array}{l}\text { SLNs Missed by } \\
\text { Radioisotope } \\
(n=394)\end{array}$} & \multicolumn{2}{|c|}{$\begin{array}{l}\text { SLNs Detected by } \\
\text { Blue Dye }(n=1628)\end{array}$} & \multicolumn{2}{|c|}{$\begin{array}{l}\text { SLNs Missed by } \\
\text { Blue Dye }(n=736)\end{array}$} & \multirow[t]{2}{*}{$p$-value } \\
\hline & Number & (\%) & Number & (\%) & Number & (\%) & Number & (\%) & Number & (\%) & \\
\hline Level III & I & 0 & I & 0.1 & 0 & 0 & 0 & 0 & I & 0.1 & - \\
\hline Level II & 196 & 8.3 & 168 & 8.5 & 28 & 7.1 & 81 & 5 & 115 & 15.6 & $<0.00 I^{*}$ \\
\hline Level I-Zone A & 680 & 28.8 & 569 & 28.9 & 111 & 28.2 & 542 & 33.3 & 138 & 18.8 & 0.099 \\
\hline Level I-Zone B & 1388 & 58.7 & 1149 & 58.3 & 239 & 60.7 & 932 & 57.2 & 456 & 62 & $<0.00 I^{*}$ \\
\hline Level I-Zone C & 99 & 4.2 & 83 & 4.2 & 16 & 4.1 & 73 & 4.5 & 26 & 3.5 & 0.164 \\
\hline
\end{tabular}

Note: ${ }^{*} p<0.05$.

Abbreviation: SLNs, sentinel lymph nodes.

in breast-conserving surgery or nipple-sparing mastectomy, surgeons may perform SLNB using the same incision as that of the lumpectomy or nipple-sparing mastectomy rather than making an additional incision in the axilla. In such settings, extended operating distance and visualization with an improper angle might make it much more difficult to track along tiny lymphatic vessels within a limited space, leading to a lower identification rate with blue dye than with radioisotope and dual tracers.

Whether prior excisional biopsy increases the incidence of missed detection has been broadly debated. While some believed that excisional biopsy may hamper the identification of SLN, other evidence suggests that it does not significantly affect the accuracy of SLNB. ${ }^{25-27}$ Intriguingly, our results indicated that prior excisional biopsy was associated with an increased identification rate of SLN using blue dye as a single tracer. This might be related to the fact that the blue dye was injected by surgeons in our institution, who would inject the blue dye intradermally at the site of incision in the direction of the axilla and intradermal/subcutaneously at the periareolar site in case of a prior excisional biopsy, avoiding the cutoff of the blue dye in lymphatic flow. However, the results should be validated in studies with larger sample sizes.

By recording the precise location of each SLN, we were able to map the distribution of SLNs based on whether they were successfully detected by the radioisotope and the blue dye. Several attempts have been made to describe the common locations of SLNs. ${ }^{28-32}$ Lo and colleagues categorized the location of the hottest spot on the skin of the axilla into seven areas divided by five landmarks. They found that $98.4 \%$ of the hotspots 
detected on the axilla skin before SLNB were located in the area demarcated by the four landmarks of the hairline, a line tangential to and $2 \mathrm{~cm}$ below the center of the hairline, the lateral border of the pectoralis major muscle, and the mid-axillary line. ${ }^{28}$ Another study designed an ellipse surrounded by the lateral line of the pectoralis major, the axillary skin fold that divides the trunk and the upper arm, and the nipple to predict the location of SLNs. $^{30}$ These two studies correlated the location of SLNs with surface markers but lacked value in guidance for practice among genuine anatomical structures during surgery. Clough and colleagues created a new anatomical classification of the lower part of the axilla based on the intersection of two anatomical landmarks, the lateral thoracic vein and the second intercostobrachial nerve. The results suggested that in $98.2 \%$ of patients, the axillary SLN was located medially, alongside the lateral thoracic vein, either below the second intercostobrachial nerve or above it. ${ }^{32}$ The above studies initially indicated the locations of SLNs. In the current study, we not only described the distribution of SLNs but also explored the locations potentially hiding SLNs detected by the radioisotope or the blue dye. The proportion of SLNs in levels II-III was $8.3 \%$, which was significantly higher than the previously reported rate of $2.3 \%$ in a study with a smaller sample size. ${ }^{33}$ More importantly, the blue dye used as a single tracer missed a notable portion of the skipped SLNs at levels II and III. We should be alert that the genuine rate of skipped SLNs might not be as low as that previously reported, and surgeons who have to apply a single tracer of the blue dye must bear in mind the SLNs in level II.

Several limitations of this study should be noted. First, the ratio of breast-conserving surgeries in this study was much lower than the international average. In the future, with more cases of lumpectomy collected, SLNBs with lumpectomy using a joint incision and that using separate incisions in the axilla and on the breast should be compared to explore the indicators for miss-detection. Second, ALND was not performed in patients with negative SLNs per the study design, and an accurate false-negative rate in our study was not calculated. Therefore, the results of this study should be validated in multicenter studies with a larger sample size.

\section{Conclusions}

The identification rate of blue dye only was lower than those of radioisotope only and dual tracers. Type of surgery, prior excisional biopsy, and number of SLNs were indicators for detection failure, and missed detection was significantly more frequent in smaller and uninvolved nodes. In terms of the location of SLNs, the blue dye tracer missed a notable portion of the skipped SLNs in level II. Therefore, surgeons who apply the blue dye as a single tracer should exercise with extra caution.

\section{Ethics Approval and Informed Consent}

The study has been approved by the institutional review board (West China Hospital Research Ethics Committee, No. 2009 [30]). Written informed consent was obtained from all individual participants included in the study, and that this study was conducted in accordance with the Declaration of Helsinki.

\section{Acknowledgments}

This study is supported by the funding from the National Natural Science Foundation of China (81902686); the Program of the Science and Technology Bureau of Sichuan (2020YJ0293); and the Post-Doctor Research Project, West China Hospital, Sichuan University (2019HXBH046).

\section{Disclosure}

The authors report no conflicts of interest in this work.

\section{References}

1. Lyman GH, Temin S, Edge SB, et al. Sentinel lymph node biopsy for patients with early-stage breast cancer: American Society of Clinical Oncology clinical practice guideline update. J Clin Oncol. 2014;32 (13):1365-1383. doi:10.1200/JCO.2013.54.1177

2. Riedel F, Heil J, Golatta M, et al. Changes of breast and axillary surgery patterns in patients with primary breast cancer during the past decade. Arch Gynecol Obstet. 2019;299(4):1043-1053. doi:10.1007/s00404-018-4982-3

3. Vermersch C, Raia-Barjat T, Chapelle C, Lima S, Chauleur C. Randomized comparison between indocyanine green fluorescence plus $99 \mathrm{~m}$ technetium and $99 \mathrm{~m}$ technetium alone methods for sentinel lymph node biopsy in breast cancer. Sci Rep. 2019;9(1):6943. doi:10.1038/s41598-019-43473-3

4. Zhou Y, Li Y, Mao F, et al. Preliminary study of contrast-enhanced ultrasound in combination with blue dye vs. indocyanine green fluorescence, in combination with blue dye for sentinel lymph node biopsy in breast cancer. BMC Cancer. 2019;19(1):939. doi:10.1186/s12885019-6165-4

5. Qin X, Yang M, Zheng X. Comparative study of indocyanine green combined with blue dye with methylene blue only and carbon nanoparticles only for sentinel lymph node biopsy in breast cancer. Ann Surg Treat Res. 2019;97(1):1-6. doi:10.4174/astr.2019.97.1.1

6. Man V, Wong TT, Suen D, Kwong A. Sentinel lymph node biopsy in early breast cancer: magnetic tracer as the only localizing agent. World J Surg. 2019;43(8):1991-1996. doi:10.1007/s00268-019-04977-1

7. Thill M, Kurylcio A, Welter R, et al. The Central-European SentiMag study: sentinel lymph node biopsy with superparamagnetic iron oxide (SPIO) vs. radioisotope. Breast. 2014;23(2):175-179. doi:10.1016/j. breast.2014.01.004 
8. Rubio I, Diaz-Botero S, Esgueva A, et al. The superparamagnetic iron oxide is equivalent to the Tc99 radiotracer method for identifying the sentinel lymph node in breast cancer. Eur J Surg Oncol. 2015;41(1):46-51. doi:10.1016/j.ejso.2014.11.006

9. Caudle AS, Yang WT, Krishnamurthy S, et al. Improved axillary evaluation following neoadjuvant therapy for patients with node-positive breast cancer using selective evaluation of clipped nodes: implementation of targeted axillary dissection. J Clin Oncol. 2016;34(10):1072-1078. doi:10.1200/JCO.2015.64.0094

10. Straver ME, Meijnen P, van Tienhoven G, et al. Sentinel node identification rate and nodal involvement in the EORTC 10981-22023 AMAROS trial. Ann Surg Oncol. 2010;17(7):1854-1861. doi:10.1245/s10434-0100945-z

11. Goyal A, Newcombe RG, Chhabra A, Mansel RE. Factors affecting failed localisation and false-negative rates of sentinel node biopsy in breast cancer-results of the ALMANAC validation phase. Breast Cancer Res Treat. 2006;99(2):203-208. doi:10.1007/s10549-006-9192-1

12. Lyman GH, Giuliano AE, Somerfield MR, et al. American Society of Clinical Oncology guideline recommendations for sentinel lymph node biopsy in early-stage breast cancer. J Clin Oncol. 2005;23 (30):7703-7720. doi:10.1200/JCO.2005.08.001

13. Cody HS, Fey J, Akhurst T, et al. Complementarity of blue dye and isotope in sentinel node localization for breast cancer: univariate and multivariate analysis of 966 procedures. Ann Surg Oncol. 2001;8 (1):13-19. doi:10.1007/s10434-001-0013-9

14. Arrington AK, Kruper L, Vito C, Yim J, Kim J, Chen SL. Rural and urban disparities in the evolution of sentinel lymph node utilization in breast cancer. Am J Surg. 2013;206(5):674-681. doi:10.1016/j. amjsurg.2013.07.007

15. Vidya R, Athwal R, Huissoon AP, Baretto RL, Krishna MT. Diagnostic application of patent blue $\mathrm{V}$ in sentinel lymph node biopsy for breast cancer-Is it time for a change? Indian J Cancer. 2019;56 (3):269-270. doi:10.4103/ijc.IJC_139_18

16. Peek MC, Kovacs T, Baker R, Hamed H, Kothari A, Douek M. Is blue dye still required during sentinel lymph node biopsy for breast cancer? Ecancermedicalscience. 2016;10(19):674. doi:10.3332/ ecancer.2016.674

17. Nori J, Vanzi E, Bazzocchi M, et al. Role of axillary ultrasound examination in the selection of breast cancer patients for sentinel node biopsy. Am J Surg. 2007;193(1):16-20. doi:10.1016/j. amjsurg.2006.02.021

18. Martin RC II, Edwards MJ, Wong SL, et al. Practical guidelines for optimal gamma probe detection of sentinel lymph nodes in breast cancer: results of a multi-institutional study. Surgery. 2000;128 (2):139-144. doi:10.1067/msy.2000.108064

19. Yang JQ, Tang SL, Zhou YT, et al. Prognostic implication of the primary tumor location in early-stage breast cancer: focus on lower inner zone. Breast Cancer. 2018;25(1):100-107. doi:10.1007/s12282017-0797-5

20. Giuliano AE, Hunt KK, Ballman KV, et al. Axillary dissection vs no axillary dissection in women with invasive breast cancer and sentinel node metastasis: a randomized clinical trial. JAMA. 2011;305 (6):569-575. doi:10.1001/jama.2011.90
21. Lyman GH, Somerfield MR, Giuliano AE. Sentinel lymph node biopsy for patients with early-stage breast cancer: 2016 American Society of Clinical Oncology clinical practice guideline update summary. J Oncol Pract. 2017;13(3):196-198. doi:10.1200/JOP. 2016.019992

22. Susini T, Nori J, Olivieri S, et al. Predicting the status of axillary lymph nodes in breast cancer: a multiparameter approach including axillary ultrasound scanning. Breast. 2009;18(2):103-108. doi:10.10 16/j.breast.2009.02.001

23. Susini T, Nori J, Vanzi E, et al. Axillary ultrasound scanning in the follow-up of breast cancer patients undergoing sentinel node biopsy. Breast. 2007;16(2):190-196. doi:10.1016/j.breast.2006.10.004

24. Gentilini O, Veronesi U. Abandoning sentinel lymph node biopsy in early breast cancer? A new trial in progress at the European Institute of Oncology of Milan (SOUND: Sentinel node vs Observation after axillary ultrasoUND). Breast. 2012;21(5):678-681. doi:10.1016/j. breast.2012.06.013

25. Wong SL, Edwards MJ, Chao C, et al. The effect of prior breast biopsy method and concurrent definitive breast procedure on success and accuracy of sentinel lymph node biopsy. Ann Surg Oncol. 2002;9 (3):272-277. doi:10.1007/BF02573065

26. Yararbas U, Argon AM, Yeniay L, Kapkac M. Problematic aspects of sentinel lymph node biopsy and its relation to previous excisional biopsy in breast cancer. Clin Nucl Med. 2009;34(12):854-858. doi:10.1097/RLU.0b013e3181becec2

27. Coskun G, Dogan L, Karaman N, Ozaslan C, Atalay C. Value of sentinel lymph node biopsy in breast cancer patients with previous excisional biopsy. J Breast Cancer. 2012;15(1):87-90. doi:10.4048/ jbc.2012.15.1.87

28. Lo C, Lee PC, Yen RF, Huang CS. Most frequent location of the sentinel lymph nodes. Asian J Surg. 2014;37(3):125-129. doi:10.1016/j.asjsur.2014.01.006

29. Ando J, Kitamura T, Kuroki Y, Igarashi S. Preoperative diagnosis of the axillary arch with multidetector row computed tomography and the axillary arch in association with anatomical problems of sentinel lymph node biopsy. Breast Cancer. 2010;17(1):3. doi:10.1007/ s12282-009-0138-4

30. Ueda N, Tada K, Miyata S, Koizumi M, Kuroda Y, Iwase T. Identification of sentinel lymph node location based on body surface landmarks in early breast cancer patients. Breast Cancer. 2009;16 (3):219-222. doi:10.1007/s12282-008-0086-4

31. Britton P, Moyle P, Benson JR, et al. Ultrasound of the axilla: where to look for the sentinel lymph node. Clin Radiol. 2010;65 (5):373-376. doi:10.1016/j.crad.2010.01.013

32. Clough KB, Nasr R, Nos C, Vieira M, Inguenault C, Poulet B. New anatomical classification of the axilla with implications for sentinel node biopsy. Br J Surg. 2010;97(11):1659-1665. doi:10.1002/ bjs. 7217

33. Marrazzo A, Palumbo VD, Marrazzo E, et al. Localization of sentinel lymph node in breast cancer. A prospective study. Int J Surg. 2014;12:S162-S164. doi:10.1016/j.ijsu.2014.05.020
Cancer Management and Research is an international, peer-reviewed open access journal focusing on cancer research and the optimal use of preventative and integrated treatment interventions to achieve improved outcomes, enhanced survival and quality of life for the cancer patient.
The manuscript management system is completely online and includes a very quick and fair peer-review system, which is all easy to use. Visit http://www.dovepress.com/testimonials.php to read real quotes from published authors. 